\title{
Concerts virtuels au chevet de patients atteints de la COVID-19 : un trio de points de vue
}

\author{
- Citation : CMAJ 2020 November 2;192:E1370-1. doi : 10.1503/cmaj.201662-f
}

Voir la version anglaise de l'article ici : Voir la version anglaise de l'article ici : www.cmaj.ca/lookup/doi/10.1503/cmaj.201662

\section{La violoncelliste}

« Ce patient atteint de la COVID-19 est malade depuis plusieurs semaines et ne va pas bien. Il est paralysé, sous sédatif et sous ventilation. Son fils souffre de cette situation et aimerait que vous jouiez de la musique pour son père. »

Je ressens le poids de cette demande, et je me connecte à sa chambre.

Silence. Le ronronnement d'une machine. Un bruissement occasionnel. Mais surtout le silence.

Avant de commencer à jouer « Sun Up », je lui demande d'imaginer le soleil se levant sur un lac paisible (annexe 1, fichier audio, accessible à www.cmaj.ca/lookup/doi/10.1503/ cmaj.201662/tab-related-content). Je veux qu'il sache qu'il s'agit d'un concert entièrement personnalisé. Même s'il ne peut pas répondre, son silence n'est pas vide. C'est tout le contraire. Je lève mon archet, j'inspire et je laisse la première note chanter avec un vibrato chaleureux. Le son porte de ma maison à son lit d'hôpital à des milliers de kilomètres de là.

Un chef d'orchestre m'a dit un jour que les silences sont les parties les plus fortes d'une composition musicale. Ils doivent être présents pour mettre en valeur la musique, pour qu'elle ait du contraste, qu'elle respire. En jouant pour ce patient, j'apprécie ce temps supplémentaire. Chaque fois que je prends une pause, je ne suis pas seule.

Deux semaines plus tard, je participe à un appel Zoom pour jouer pour le même patient. II n'est plus sous ventilateur, mais il a subi une trachéotomie. À ma grande surprise, 16 autres cases peuplent l'écran. La famille, les amis, les médecins et les infirmières entourent le patient en ligne. Nous découvrons un moment privilégié, bien que virtuel, d'humanité partagée. Je

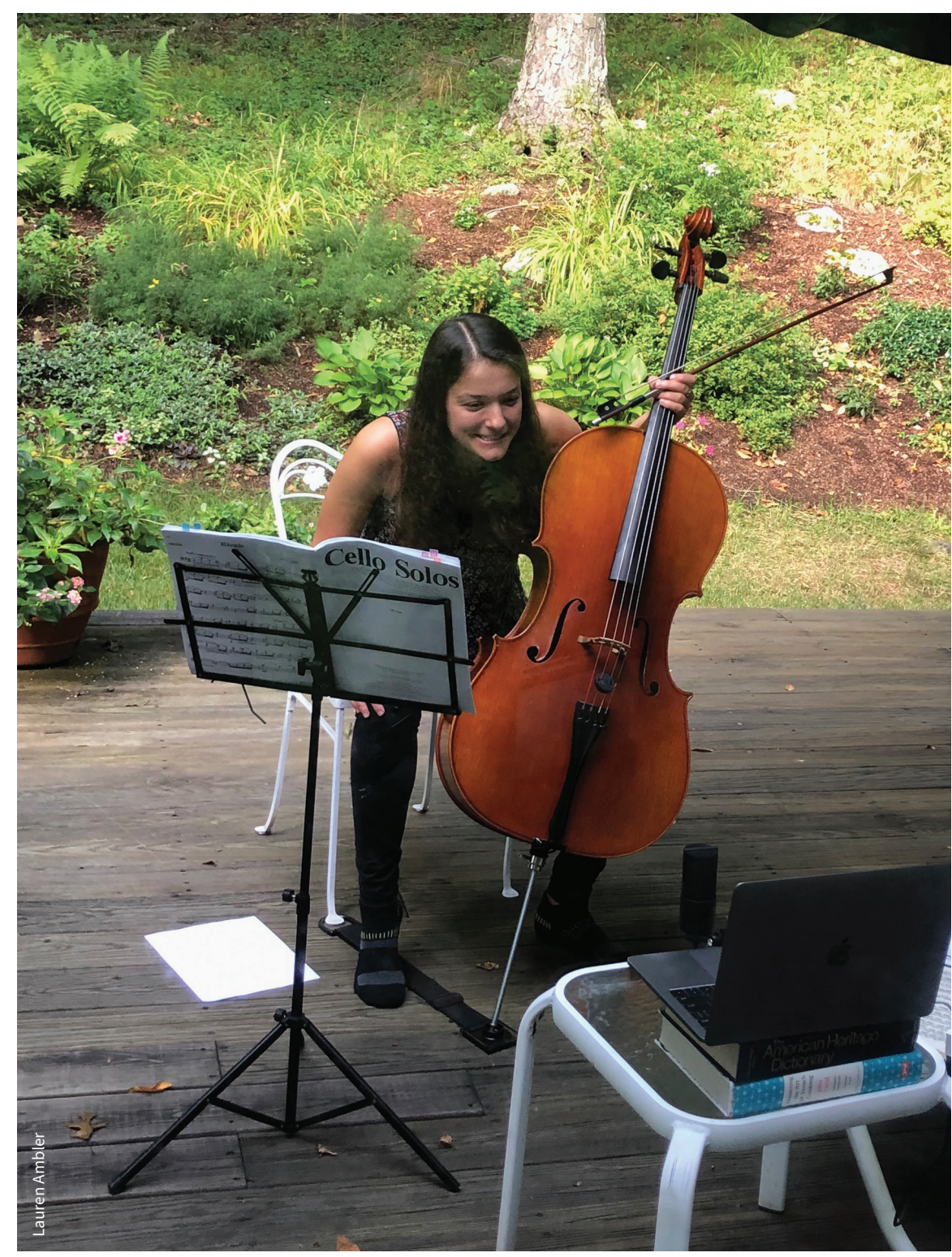

Melanie Ambler se prépare à jouer devant son public virtuel.

plonge dans mon violoncelle comme jamais auparavant, je souris et je reconnais toute la joie, l'optimisme, et la chance que j'ai d'être en bonne santé.
Je parle à travers ma musique et j'interagis avec un public entièrement présent, mais muet. Cette occasion intime de jouer pour des étrangers dans leur situation la 
plus vulnérable m'émeut plus que toute autre performance que j'ai donnée. Je partage des pièces qui me tiennent à cœur. Ce n'est pas comme faire jouer un enregistrement. Nous connectons à travers la musique. Nous respirons ensemble.

\section{L’infirmière}

«Passons en revue les patients. Lequel pourrait bénéficier d'un concert en direct? »

Le choix est difficile, tant de patients sont sous ventilation et reçoivent une sédation. Beaucoup ne se réveillent pas comme les autres patients que nous avons soignés dans le passé. L'une d'entre elles est avec nous depuis des semaines, sous ventilation, complètement isolée de sa famille et de ses amis. Une musique apaisante peut-elle pénétrer le brouillard de la sédation? Elle doit se trouver là, quelque part.

J'appelle la violoncelliste.

Elle sourit, impatiente de jouer. "Je sais que vous êtes occupée. Vous pouvez laisser la tablette dans la chambre de la patiente si vous le souhaitez, et je me déconnecterai lorsque j'aurai fini de jouer. »

Ouf! J'ai tellement de choses à faire.

J'entre dans la chambre cacophonique, et je vois sur la tablette la violoncelliste prête à jouer. Je m'assieds près du lit de la patiente et lui tiens la main. J'ai tenu tant de mains au cours des derniers mois. Je songe à toutes ces familles qui ont vu par le truchement de cette même tablette leurs proches rendre leur dernier souffle, pendant que je leur tenais la main; une situation trop fréquente pour une infirmière des soins intensifs de l'unité COVID-19. La violoncelliste joue de son bel instrument et je commence à mieux respirer, comme si je ne portais pas ce masque N95.

Je ferme les yeux et trouve du réconfort dans la musique. L'infirmière principale de la patiente écoute avec moi. La violoncelliste peut penser qu'elle n'offre le cadeau de la musique en direct qu'à la patiente, mais pendant cette période de pertes et de stress inimaginables, elle nous donne une lueur d'espoir. Nous lui en sommes reconnaissantes. Nous fixons la patiente, en espérant que ses yeux s'ouvriront. Elle a besoin de se rétablir. Elle est comme nous. Cette patiente est une infirmière.

\section{Le médecin}

Le patient respire à un rythme de 40 respirations par minute. Il se réveille lentement après 7 semaines de soins intensifs, sous ventilation, la plupart du temps sous sédation. Maintenant, avec une trachéotomie et une très grande faiblesse, il passe de moments de lucidité et de stabilité à d'autres de confusion et d'anxiété. Lorsque la violoncelliste, à des milliers de kilomètres de là, joue sa première note, ses yeux se déplacent vers l'écran. Sa fréquence respiratoire descend à 20 respirations par minute. Il est calme et concentré.

Avec leur téléphone cellulaire pour se connecter au concert, l'équipe de médecins résidents, qui travaille sans relâche pour ce patient, et tant d'autres, se rassemble avec des bols de crème glacée à l'extérieur de la pièce. Ils m'invitent à me joindre à eux. Inspiré par la respiration détendue du patient, je rejoins l'équipe et je respire à mon tour pendant quelques minutes. Alors que la musique joue, un résident lance spontanément : «Ce travail est si difficile».

Oui, ce travail est si difficile. Voir les gens dans leur situation la plus vulnérable. Voir la joie et la tristesse qui accompagnent le parcours de chaque patient qui arrive dans le lit devant nous. Et maintenant, pendant la pandémie de COVID-19, de savoir que nous sommes les seuls à pouvoir les accompagner dans les moments les plus difficiles de leur vie. Il est impossible de se préparer à l'expérience de pleurer avec une famille lors d'une vidéoconférence alors que nous tenons la tablette pour qu'elle puisse dire au revoir à son proche, ou de partager la joie de libérer une autre personne du joug de la ventilation artificielle.

Au son du violoncelle, notre équipe explore les douleurs et les plaisirs d'être médecin en ce moment. Alors que la musique atteint un crescendo, elle porte nos émotions jusqu'à la surface. Puis un legato apaisant survient et nous découvrons l'amplitude de ce dont nous sommes témoins au quotidien. À travers les larmes, les moments de silence et la crème glacée, nous discutons de ce qui nous est venu à l'esprit, mais qui est rarement reconnu en première ligne. Nous nous demandons si tout ira bien. Bien que je ne connaisse pas la réponse à cette question, alors que la violon- celliste entame son dernier morceau, nous écoutons et respirons ensemble. J'ai espoir.

\section{Melanie Ambler MSc}

Université Brown, Providence, RI

\section{Laura Wodecki IA MSci}

Rhode Island Hospital/Lifespan Health

System, Providence, RI

\section{Timothy Amass MD MSc}

Division des sciences pulmonaires et de la médecine des soins intensifs, École de médecine de l'Université du Colorado; Department of Veterans Affairs, Système de soins de santé de l'Est du Colorado, Denver, CO

\section{Référence}

1. Weiser B. These are the bedside concerts comforting virus patients. The New York Times 2020 May 3 Accessible ici : www.nytimes.com/2020/05/03/ nyregion/coronavirus-doctor-musician-rachel -easterwood.html (consulté le 8 juillet 2020).

Cet article a été révisé par des pairs.

L'un des patients a donné son consentement pour que cette histoire soit racontée; la fille de l'autre a donné son consentement.

Les auteurs ont obtenu l'autorisation d'utiliser le fichier audio "Sun Up », composé en 2008 par les violoncellistes Stephen Katz et Derek Snyder. Cette pièce était à l'origine pour 6 violoncelles.

Propriété intellectuelle du contenu : Il s'agit d'un article en libre accès distribué conformément aux modalités de la licence Creative Commons Attribution (CC BY-NC-ND 4.0), qui permet l'utilisation, la diffusion et la reproduction dans tout médium à la condition que la publication originale soit adéquatement citée, que l'utilisation se fasse à des fins non commerciales (c.-à-d., recherche ou éducation) et qu'aucune modification ni adaptation n'y soit apportée. Voir : https://creativecommons. org/licenses/by-nc-nd/4.0/.

Remarque de l'auteur : Récemment, le New York Times a publié un article décrivant comment la $D^{\text {re }}$ Rachel Easterwood, intensiviste à l'Hôpital New York-Presbyterian Allen, a commencé à coordonner des concerts virtuels au chevet de ses patients atteints de la COVID-19 en isolement obligatoire ${ }^{1}$. Inspirée par cette histoire, une violoncelliste et future médecin, Melanie Ambler, a contacté le personnel de l'hôpital, lui demandant si elle pouvait partager sa musique avec des patients gravement malades. Melanie Ambler a mis en place le projet musICforU et a reçu le prix Rosalind-Swenson-Enrichment de la Commission franco-américaine pour lancer un programme de concerts virtuels en direct dans les hôpitaux et les foyers de soins de longue durée en France, appelé Petite pause musicale. Tous les hôpitaux intéressés par des concerts virtuels en direct sont invités à la contacter à l'adresse petitepausemusicale@gmail.com. 